The proximal end of the tube is brought out through the mouth and fixed with adhesive plaster to the side of the cheek. The distal end is anchored to the skin at the site of the incision. Vaseline packing is gently tucked into the incision, and the tube is occluded by a clamp at its distal end. If the condition has had a dental origin the extraction of the tooth involved completes the operation.

\section{Post-operative Care}

Chemotherapy in the form of sulphonamide drugs is used as a routine auxiliary treatment. Gas-forming organisms often predominate in the infection, and in at least two of our cases gas under tension has escaped on incising the deep cervical fascia. We have therefore used hydrogen peroxide $1 / 10$ as an irrigant. It seems likely that zinc peroxide suspension would be more efficacious, but its availability is limited. During the patient's waking hours there is a continuous slow drip of irrigating solution introduced through the proximal end of the tube. This escapes from the openings in the tube and allows the region to be gently irrigated throughout its various layers (Shepherd, 1940). The fluid coming out of the incision around the sides of the tube presents some difficulties, as it tends to drip down the side of the neck, but with diiigent and zealous nursing care it may be collected in a kidney dish hung on the patient's neck, or a close-fitting jaconet collar.

When continuous irrigation has not been convenient we have compromised with $100-\mathrm{c} . \mathrm{cm}$. irrigations every four hours, after which the clamp is removed and the tube functions as an ordinary through-and-through drain. After 48 hours the general and local improvement is sufficient to consider shortening the tube. It is cut away flush with the buccal mucosa and shortened about half an inch. If further irrigations are indicated at this stage, they are most easily carried out by introducing a small catheter beside the drainage-tube and syringing hydrogen peroxide through it. The drainage-tube is removed on the fourth to sixth post-operative day, and a vaseline dressing, changed when necessary, completes the post-operative dressings.

Although all these incisions have healed by slow granulation and subsequent epithelization, a linear scar results, and no cases have required excision for cosmetic reasons.

\section{Summary}

Possible reasons are outlined for the relatively high incidence of Ludwig's angina among East African native troops.

The diagnostic signs that make surgical intervention imperative are stated.

A system of management is described effecting free drainage and submandibular decompression followed by a drip irrigation.

We wish to record our thanks to Brig. R. P. Cormack, O.B.E., D.D.M.S., East Africa Command, and Col. D. Bell, O.B.E., E.A.A.M.C., for permission to publish this paper; and to acknowledge the co-operation of S/Sgt. F. Finch, R.A.M.C., for the production of the accompanying diagrams.

\section{REFERENCES}

Fry, W. Kelsey, Shepherd, P. R., McLeod, A. C., and Parfitt, G. J. (1942) The Dental Treatment of Maxillo-Facial Injuries, Blackwell Scientific Publications, Oxford

Kekwick, A., and Wright, J. (1945). (Not yet published.)

Shepherd, P. R. (1940). Brit. dent. J., 69, 430 .

\section{INGUINAL HERNIA IN THE MERCHANT NAVY AN ANALYSIS OF 200 CONSECUTIVE CASES \\ BY}

PERCIVAL P. COLE, F.R.C.S.

E.M.S. and Senior Surgeon, Dreadnought Hospital

The incidence and cure of hernia have attracted renewed and critical attention from the point of view particularly of the associated disability in men of the fighting Forces. Detailed observation and docketed conclusions have been made possible by the concentration of large numbers of men under an organized and centralized medical service. Inguinal hernia has been made the subject of a critical review by Brig. Harold Edwards (1943), who deals with it comprehensively but also with special reference to Army statistics and military considerations. In the Army; selection of cases for operation is determined by the answer to the question whether operation will make the man a more efficient soldier. In the Merchant Navy-not ranked as one of the fighting Services-the question is the more vital one of going to sea or not.

\section{Classification}

To emphasize particularly the age at operation, the large number of cases requiring supplementary repair, the largest portion of direct hernias, and the early age at which direct hernia occurs, the 200 cases under review have been classified numerically into the following groups:

Type of Hernia.-Oblique, 97; direct, 103.

Age at Operation.-53 cases were over 45, 38 were over 50, 19 were over 55 , and 9 were over 60 .

Recurrent hernias dealt with in this series numbered 22 . Of these, 18 were direct and 4 were oblique. Four of these recurrences followed a previous filigree operation, and occurred thereafter: 1 at 10 months, 1 at 6 years, 1 at 12 years, 1 at 25 years.

Primary direct hernia occurring at the age of 40 and under, 27 cases. Earliest age at which direct hernia was found, 23 years.

Number of cases dealt with by filigree repair, 130. The first operation was performed on Feb. 19, 1941, and the last on June 26, 1944.

For the purpose of comparison with procedure in the recognized fighting Services a brief analysis will be based upon that embodied in an Army Medical Department Bulletin on Inguinal Hernia, issued by the War Office in Feb., 1943. Therein it is stated that over $12 \%$ of inguinal hernias recur after operation, and this recurrence rate is regarded as representative of British surgery as a whole; the factors concerned are considered to be: (1) surgical skill and experience of the operator ; (2) selection of cases for operation; (3) operative detail ; (4) post-operative management.

\section{Analysis of Cases}

1. In connexion with this factor all the cases have been operated upon either by me or under my direct supervision.

2. Selection of Cases for Operation.-Under this heading the Memorandum excludes the following categories as being unsuitable: (a) small bulges in men of inferior abdominal musculature ; $(b)$ large scrotal hernias in pot-bellied subjects of poor physique; (c) large recurrent hernias in similar subjects; (d) large hernias in men of low category because of other disorders. Among the 200 cases on which this analysis is based will be found instances included in the first three categories, and, in fact, discrimination on these lines has not been exercised, for it is a cardinal principle that no case is rejected on account of the local condition. With regard to category $d$, I am in agreement with this reason for rejection, but actually no such case has been met with. As I have previously stated (Cole, 1941), the operability rate in my hands, taking all cases into consideration, is $90 \%$ at a modest estimate.

3. Operative Detail.-Under this heading it is stated that $75 \%$ of recurrences after operation for indirect hernia were indirect. Authorities such as Page (1934), Block (1933), Ogilvie (1937), and Gallie and Le Mesurier (1924) have agreed that by far the greatest number of so-called recurrences after operation for oblique hernia are direct in form. It is clear that in this mass reproduction of the original condition $(75 \%)$ responsibility must be attributed to factors either non-operative or operative to a minimal extent in the collected series of the quoted authorities. Prior to the series under survey, and over a period of 20 years, recurrent hernias numbering approximately 400 have been observed, and the mode of recurrence has been so often direct that this predominance has never been questioned. The small number of recurrences in the present series bears this out, for it will be observed that of the 22 recurrences included 18 were direct and only 4 oblique. Recurrences, the Memorandum states, are commonest after the Bassini operation or one of its modifications. With this my own practice records agreement, for I have not performed a Bassini operation for many years. I further agree with the recommendation that "no type of operation which interferes with the normal muscular apparatus of the inguinal canal should be practised for uncomplicated indirect hernia in the young soldier"; and in this connexion it is the age of the patient that counts and not the nature of his employment.

Apart from this simple type of hernia occurring in the young man, some supplementary method must be employed to replace 
or reinforce the failed inguinal canal. This applies to the long-standing oblique hernia, for the control of which a truss has frequently been employed for varying periods up to several years, and to all cases of direct hernia.

The supplementary measures recommended for use in the Army involve the employment of fascia in various ways. The supplementary procedure adopted in this series of cases is that of silver filigrees. No case has been subjected to this procedure unless the indications have been perfectly clear. In other words, 130 of the 200 cases have been of such a nature that cure could not have been expected without resort to this or some other method of supplementary repair.

Textbooks are in substantial agreement that the treatment of direct hernia is modified by the fact that this type of lesion usually occurs in elcerly men. I believe that direct hernia at earlier ages occurs more frequently in men of the Merchant Navy than in other walks of life. Whether this be so or not, it is significant that of the 200 cases operated upon 103 have belonged to this category, that 27 of these have come to operation at or under the age of 40 , and that direct hernia has been found at the early age of 23 . In connexion with the question of age, it is noteworthy that 53 hernias in this consecutive series occurred in men of over 45. I suggest that in none of the three recognized fighting Services would men of such an age apply or be referred for operation with the sole objective of resuming or maintaining their active and hazardous participation in the prosecution of the war.

Sepsis.-The Memorandum previously cited refers to "sepsis in hernia wounds." "Sepsis," it states, "is relatively common." In this consecutive series sepsis has been entirely absent except for an occasional transient superficial affection of the skin margins. I attribute this to the fact that the so-called nontouch technique has been the ideal aimed at. Skin towels are invariably used, handling of the tissues in the wound is reduced to a minimum and in most cases rigidly excluded. This technique has been facilitated by the routine use of the centraleyed or double-pointed needle (Cole).

4. Post-operative Management of Fiiigree Cases.-Following a filigree operation patients are confined to bed for three weeks. Normal activities are gradually resumed until full work is undertaken at the end of four months. No systematic post-operative exercises have been prescribed.

\section{End-results}

Return to Duty and Morbidity.-It is significant that all cases operated upon have been returned to the reserve pool, and, so far as I know, have resumed their duty at sea. Morbidity even has been negligible. In only one case was return to duty delayed beyond the normal period. This was due to the occurrence of femoral thrombosis on the opposite side some weeks after operation.

Recurrences.-Of the 22 recurrences in this series four occurred, as noted, after a primary filigree operation. Repair in each case was by additional filigree, with uniform success and return to work.

Filigree Recurrences in the Merchant Navy.-In the 10-year period $1930-9,202$ recurrences were met with out of a total of 2.015 operations, and of these recurrences only 12 followed a filigree operation. The number of filigree operations performed in this period was 448 . The filigree operation has been largely confined to the Dreadnought Hospital for' many years, and it is not unreasonable to suppose that cases of recurrence following its performance would return, be returned, or be reported. It might be suggested that the 12 recurrences met with in 1930-9 were partly applicable to a preceding period when few such operations were undertaken. This objection is answered by the fact that in the preceding five years-1925-9 -287 cases were dealt with by this method. In view of the high operability rate and of the fact that the operation is reserved for cases that demand some supplementary method of repair, a high group incidence of recurrence might well be expected. The figures cited clearly suggest that the recurrence rate attaching to this operation is remarkably low.

The method of filigree repair has been accorded a recognized place in current surgical practice in the pages of the most recent-third-edition of Modern Operative Surgery, and the editor, Prof. Grey Turner, in a short historical survey states that "the lapse of time is showing that this plan has attained more success than has been acknowledged."

Details of the technique will be found in an article contributed by me to the British Journal of Surgery (Cole, 1941).

\section{Illustrative Cases}

1. Second Officer, aged 27. Six months' history. Right inguinal hernia. Operation March, 1942. Small direct bulge. Repaired by two filigrees.

2. Navigating Officer, aged 27. Right inguinal hernia gradually coming on for six years, with more rapid increase lately. Operation Nov. 16, 1942. Conjoined tendon very poorly represented. Hernia presented as diffuse direct bulge. Deep epigastrics tied. Repaired by two filigrees.

3. A big stout man, aged 60 . Irreducible left inguinal hernia Operation June 16, 1941. Sliding hernia of the sigmoid. Repaired by two filigrees. Thereafter hernia developed on right side (a tribute, surely, to the efficacy of the left-sided repair). Operation June 26, 1942. Direct bulge. Repaired by two filigrees.

4. Master, aged 52 . Weight 12 st. $8 \mathrm{lb}$. Saddlebag type of sac. Diffuse direct bulge of the posterior wall, continued behind epigastric vessels into the cord. Epigastric vessels tied. Repaired by two filigrees.

5. Quartermaster, aged 54. Left inguinal hernia of 10 years' duration; right inguinal hernia of 9 years' duration. Wearing truss for 9 years. Operation Aug. 31, 1942: Direct left inguinal hernia repaired by two filigrees. Sept. 14, 1942: Direct right inguinal hernia repaired by two filigrees.

6. Steward, aged 51. Six months' history. Chronic cough. Right inguinal hernia. . Operation June, 1942. Direct bulge. Lipoma of cord excised. Repaired by two filigrees.

7. Tug driver on the Thames by night and day, aged 59. A big stout man of 14 st. Right inguinal hernia for 20 years, at first reducible, but lately becoming irreducible. Operation only alternative to giving up his employment. Operation Nov. 27, 1941. Large oblique sac to which omentum was adherent and of which $4 \frac{1}{2} \mathrm{lb}$. was ligatured and removed. Repaired by two filigrees. Has gained over 2 st. -16 st. $8 \mathrm{lb}$.- and has forgotten about his hernia. In a letter received Aug. 25, 1944, he states: "I have never felt better. I am still at work, and have not lost a day's work since you discharged me. I have felt no effects of my hernia." This has been confirmed since by personal contact.

\section{REFERENCES}

Army Medical Department Bulletin, Feb., 1943. Supp. No. 4.

Block, W. (1933). Arch. klin. Chir., 175, 607; 176, 812.

Cole, P. P. (1941). Brit. J. Surg., 29, 168.

Gallie, W. E., and Le Mesurier, A. B. (1924). Ibid., 12, 289.

Ogilvie, W. H. (1937). Proc. roy. Soc. Med., 30, 532.

Page, C. Max (1934). British Medical Journal, 2, 896

\section{Medical Memoranda}

\section{A Fatal Case of Tetanus despite Toxoid}

Active immunization against tetanus was introduced into the Army in 1939, Boyd (1938) doing the preliminary work. The present practice is to give a primary inoculation of two $1-\mathrm{c} . \mathrm{cm}$. doses of tetanus toxoid spaced six. weeks apart, and $1 \mathrm{c.cm}$. thereafter yearly as a recall dose. All available figures (Bensted, 1940 ; Smith, 1941) confirm that good protection is given thereby, but individual exceptions may occur as in the comparable immunization against diphtheria. Knowledge of the duration of protection rests at present, however, almost wholely on laboratory studies. A number of non-fatal cases of tetanus despite toxoid have been described, but reported fatal cases are rare (Boyd and Maclennan, 1942; McGill, 1943; Norman, 1943).

\section{CASE REPORT}

An Anglo-Indian aged 40 came under treatment on Feb. 7, 1944 for prolapsed, inflamed, and ulcerated piles. These were ligatured and excised ten days later under stovaine analgesia. On Feb. 26, seven days after the operation, uncontrollable jerking of the legs began, and on Feb. 29 spasms of the abdominal muscles and fever followed. Next day the neck and jaw were stiff, and infrequent short opisthotonic spasms began. On March 2 fully developed tetanus was present, with severe spasms, each lasting a second or two, and recurring every half-minute or less.

Specific treatment consisted of $1 \mathrm{c} . \mathrm{cm}$. of tetanus toxoid, and of $1,010,000$ i.u. of serum spread over the next four days. Avertin and intravenous nembutal were used symptomatically. He died on the eighth day of the disease (March 5), and, apart from bronchopneumonia, the necropsy revealed nothing. The operation wounds 